\title{
ESTIMATION OF THE POWER PEAKING FACTOR IN A NUCLEAR REACTOR USING SUPPORT VECTOR MACHINES AND UNCERTAINTY ANALYSIS
}

\author{
IN HO BAE, MAN GYUN NA*, YOON JOON LEE ${ }^{1}$ and GOON CHERL PARK ${ }^{2}$ \\ Department of Nuclear Engineering, Chosun University \\ 375 Seosuk-dong, Dong-gu, Gwangju 501-759, Korea \\ ${ }^{1}$ Cheju National University, 1 Ara-il-dong, Jeju-do 690-756, Korea \\ ${ }^{2}$ Seoul National University, 599 Gwanak-ro, Gwanak-gu, Seoul 151-742, Korea \\ "Corresponding author. E-mail : magyna@chosun.ac.kr \\ Received December 24, 2008 \\ Accepted March 30, 2009
}

Knowing more about the Local Power Density (LPD) at the hottest part of a nuclear reactor core can provide more important information than knowledge of the LPD at any other position. The LPD at the hottest part needs to be estimated accurately in order to prevent the fuel rod from melting in a nuclear reactor. Support Vector Machines (SVMs) have successfully been applied in classification and regression problems. Therefore, in this paper, the power peaking factor, which is defined as the highest LPD to the average power density in a reactor core, was estimated by SVMs which use numerous measured signals of the reactor coolant system. The SVM models were developed by using a training data set and validated by an independent test data set. The SVM models' uncertainty was analyzed by using 100 sampled training data sets and verification data sets. The prediction intervals were very small, which means that the predicted values were very accurate. The predicted values were then applied to the first fuel cycle of the Yonggwang Nuclear Power Plant Unit 3. The root mean squared error was approximately $0.15 \%$, which is accurate enough for use in LPD monitoring and for core protection that uses LPD estimation.

KEYWORDS : Local Power Density, Power Peaking Factor, Subtractive Clustering, Support Vector Machine

\section{INTRODUCTION}

The monitoring of detailed 3-Dimensional (3D) core power distribution is a prerequisite for the operation of nuclear power reactors to ensure that various safety limits imposed on the fuel pellets and fuel clad barriers, such as the Local Power Density (LPD) and the Departure from the Nucleate Boiling Ratio (DNBR), are not violated during the reactor's operation.

The LPD and DNBR need to be calculated in order to perform the two major functions of the Core Protection Calculator System (CPCS) and the Core Operation Limit Supervisory System (COLSS) [1], each of which plays a role in the protection and monitoring systems of the Optimized Power Reactor 1000 (OPR1000) and the Advanced Power Reactor 1400 (APR1400). After the CPCS calculates safety-critical parameters, such as the LPD and the DNBR, it protects a nuclear reactor by tripping the reactor when its operating limits are exceeded. On the other hand, after the COLSS calculates the parameters of interest, such as the LPD and the DNBR, by using algorithms that are different from those of the CPCS, it helps plant operators to monitor the Limiting Conditions for Operation (LCOs) specified in the technical specifications.

The LPD needs to be estimated accurately to prevent nuclear fuel rods from melting. The LPD at the hottest part of a hot fuel rod, which is related to the Power Peaking Factor (PPF, $F_{q}$ ), is more important than the LPD located at any other position in the reactor core. On-line monitoring techniques that use artificial intelligence and its applications to the nuclear engineering field have been explained and reviewed by Garvey et al. [2] and Heo [3]. A lot of research [4-12] has been performed to calculate safety-critical parameters, such as the DNBR and the LPD, by using artificial intelligence methods that have been extensively used in a variety of engineering problems.

Support Vector Machines (SVMs) have been applied to classification problems. However, along with the introduction of Vapnik's $\varepsilon$-insensitive loss function [13], SVMs have also been extended and been widely used to solve nonlinear regression estimation problems. In SVM regression, the input data is mapped onto a high dimensional feature space, and subsequently, the linear regression is carried out in the feature space. 
The objective of this paper is to predict the PPF in a reactor core by using measured signals of the reactor coolant system and by applying SVMs according to the operating conditions. A quantitative analysis was also conducted to predict the uncertainty. This paper partly deals with the regression model by using SVMs to estimate the LPD, which has been the subject of prior research [12]. Furthermore, this paper builds on prior research by analyzing the uncertainty of the PPF estimates.

The output and input data employed in this study were the PPF values in the reactor core and numerous measurement signals, which are characterized by the reactor power, core inlet temperature, pressurizer pressure, coolant flowrate of the reactor core, Axial Shape Index (ASI), a variety of control rod positions, and incore neutron detector signals. The PPF values in the reactor core were estimated by the developed SVM models by using these various measurement data as the input to the SVM models. The proposed PPF estimation algorithm was verified by using nuclear and thermal data acquired from many numerical simulations of the Yonggwang Nuclear Power Plant Unit 3 (YGN-3).

\section{A REGRESSION MODEL THAT USES SUPPORT VECTOR MACHINES}

SVMs are learning systems that use the hypothesis space of linear functions in a high dimensional feature space. They are optimized with a learning algorithm that originates from the theoretical foundations of statistical learning theory and Structural Risk Minimization (SRM). Artificial Neural Networks (ANNs) use a traditional Empirical Risk Minimization (ERM) principle to minimize the estimation errors on the training data [14]. On the other hand, SVMs use an SRM principle to minimize the upper bound on the expected risk, which is the sum of the empirical risk and of the confidence interval [15]. The different method of risk minimization used in SVMs leads to a better generalization performance of SVMs compared with ANNs [15]. SVMs can be well applied to regression and classification problems.

An SVM is given $N$ training data points $\left\{\left(\mathbf{x}_{i}, y_{i}\right)\right\}_{i=1}^{N} \in$ $R^{m} \times R$, where $\mathbf{x}_{i}$ is the input vector to the SVM, and $y_{i}$ is the actual output value, from which it learns an inputoutput relationship. The SVM regression model can be expressed as follows [16]:

$$
y=f(\mathbf{x})=\sum_{i=1}^{N} w_{i} \phi_{i}(\mathbf{x})=\mathbf{w}^{r} \boldsymbol{\varphi}(\mathbf{x})+b
$$

Equation (1) is a nonlinear regression model because the resulting hyper-surface is a nonlinear surface that hangs over the $m$-dimensional input space. However, after the input vectors $\mathbf{x}$ are mapped into the vectors $\varphi(\mathbf{x})$ of a high dimensional kernel-induced feature space, the nonlinear regression model is turned into a linear regression model in the feature space. Therefore, a nonlinear function is learned by using a linear learning machine of which the learning algorithm minimizes a convex functional. The convex functional is expressed as the following regularized risk function, and the parameters $\mathbf{w}$ and $b$ are the support vector weight and bias that are calculated by minimizing the risk function:

$$
R(\mathbf{w})=\frac{1}{2} \mathbf{w}^{T} \mathbf{w}+\lambda \sum_{i=1}^{N}\left|y_{i}-f(\mathbf{x})\right|_{i},
$$

where

$$
\left|y_{i}-f(\mathbf{x})\right|_{\varepsilon}= \begin{cases}0 & \text { if }\left|y_{i}-f(\mathbf{x})\right|<\varepsilon \\ \left|y_{i}-f(\mathbf{x})\right|-\varepsilon & \text { otherwise }\end{cases}
$$

The regularized risk function of Eq. (2) consists of two terms: the first term is a weight vector norm and the second term is an estimation error. The two design parameters for the SVM regression model are the insensitivity zone $\varepsilon$ and the regularization parameter $\lambda$. The regularization parameter determines the trade-off between the estimation error and the weight vector norm. An increase in the regularization parameter penalizes larger errors, which leads to a decrease in the estimation error. This can also be achieved easily by increasing the weight vector norm. However, an increase in the weight vector norm does not ensure good generalization of the SVM regression model. This generalization property is of particular interest to data-based model development because a good model is not a model that performs well on only training data, but a model that performs well even on other data which is not training data.

$\left|y_{i}-f(\mathbf{x})\right|_{\varepsilon}$ is called the $\varepsilon$-insensitive loss function [15]. The loss is equal to zero if the predicted value, $f(\mathbf{x})$, falls within the insensitivity zone. For all predicted points outside the insensitivity zone, the loss is equal to the magnitude of the difference between the predicted value and the insensitivity zone boundary [refer to Fig. 1]. Increasing the insensitivity zone means a reduction in the requirements for the accuracy of the estimation and a decrease in the number of Support Vectors (SVs), leading to data compression. In addition, as seen in Fig. 1, increasing the insensitivity zone has smoothing effects on the modeling of highly noisy polluted data.

The regularized risk function of Eq. (2) is converted into the following constrained risk function:

$$
R\left(\mathbf{w}, \xi, \xi^{*}\right)=\frac{1}{2} \mathbf{w}^{T} \mathbf{w}+\lambda \sum_{i=1}^{N}\left(\xi_{i}+\xi_{i}^{*}\right)
$$




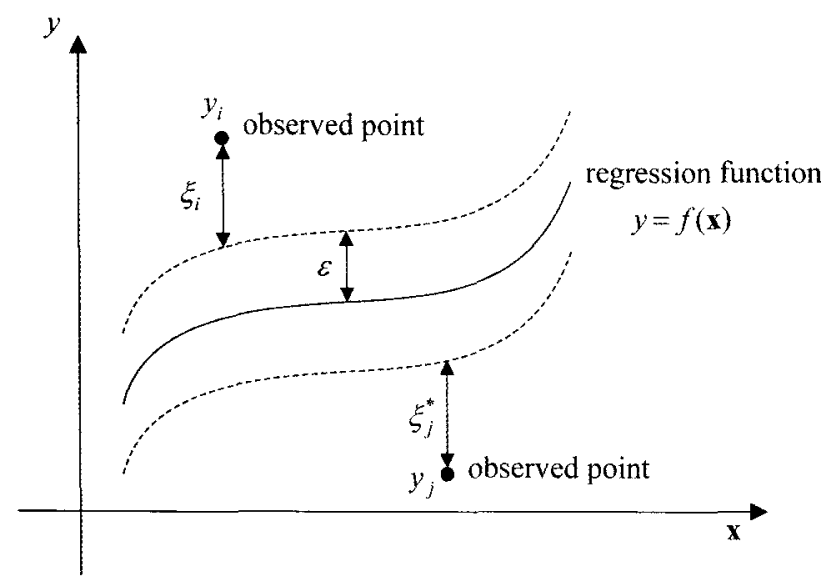

Fig. 1. Parameters for the SVM Model [21]

subject to the constraints $\begin{cases}y_{i}-\mathbf{w}^{T} \boldsymbol{\varphi}(\mathbf{x})-b \leq \varepsilon+\xi_{i}, & i=1,2, \cdots, N \\ \mathbf{w}^{T} \boldsymbol{\varphi}(\mathbf{x})+b-y_{i} \leq \varepsilon+\xi_{i}^{*}, & i=1,2, \cdots, N \\ \xi_{i}, \xi_{i}^{*} \geq 0, & i=1,2, \cdots, N\end{cases}$

where

$$
\begin{aligned}
& \xi=\left[\begin{array}{llll}
\xi_{1} & \xi_{2} & \cdots & \xi_{N}
\end{array}\right]^{r}, \\
& \xi^{*}=\left[\begin{array}{llll}
\xi_{1}^{*} & \xi_{2}^{*} & \cdots & \xi_{N}^{*}
\end{array}\right]^{T} .
\end{aligned}
$$

The parameters $\xi_{i}$ and $\xi_{i}^{*}$ are the slack variables that represent the upper and lower constraints on the output of the system, respectively, and are positive values [refer to Fig. 1].

The constrained optimization problem of Eq. (4) can be solved by applying the Lagrange multiplier technique to Eqs. (4) and (5), and by using a standard quadratic programming technique. Finally, the regression function of Eq. (1) becomes

$$
y=f(\mathbf{x})=\sum_{i=1}^{N} \beta_{i} \varphi^{T}\left(\mathbf{x}_{i}\right) \varphi(\mathbf{x})+b=\sum_{i=1}^{N} \beta_{i} K\left(\mathbf{x}, \mathbf{x}_{i}\right)+b
$$

where $K\left(\mathbf{x}, \mathbf{x}_{i}\right)=\varphi^{T}\left(\mathbf{x}_{i}\right) \varphi(\mathbf{x})$ is known as the kernel function, and the coefficient $\beta_{i}$ is a function of the Lagrange multipliers $\alpha_{i}$ and $\alpha_{i}^{*} ; \beta_{i}=\alpha_{i}-\alpha^{*}$. In this paper, the SVMs use the following radial basis kernel function:

$$
K\left(\mathbf{x}, \mathbf{x}_{i}\right)=\exp \left(-\frac{\left(\mathbf{x}-\mathbf{x}_{i}\right)^{T}\left(\mathbf{x}-\mathbf{x}_{i}\right)}{2 \sigma^{2}}\right)
$$

A lot of the coefficients $\beta_{i}$ are nonzero values, and the training data points that correspond to the nonzero values, which are known as $\mathrm{SVS}$, have an estimation error that is greater than or equal to the insensitivity zone.

\section{UNCERTAINTY ANALYSIS}

When SVM regression models are used to estimate safety-critical parameters, such as the LPD, the model estimates require an uncertainty analysis to determine how accurate the predictions of the data-based models are. Through an uncertainty analysis, a prediction interval can be calculated such that the exact value exists in the prediction interval at a specified confidence level.

There are several possible sources of uncertainty in predictions that use data-based models. This includes selection of training data, model structure that includes complexity, and noise in the input variables and the output variables [17]. Since an SVM model is developed by using a given training data set, each possible training data set that is selected from the entire population of data will generate a different model, resulting in a distribution of predictions for a given observation. Model misspecification may also take place when a model structure is not correct, thereby introducing a bias.

\subsection{Bootstrap Method}

The bootstrap method works by generating many bootstrap samples of the training data set and by retraining the SVM model parameters on each bootstrap sample. After repetitive sampling and training, the resulting predictions can provide a distribution for the LPD value. This distribution can be used to calculate prediction intervals. There are two general algorithms for the bootstrap method: bootstrap pairs sampling and bootstrap residual sampling. In this paper, the bootstrap pairs sampling algorithm was used. The available data were divided into development data and test data. The development data consisted of a large pool of data from which training and verification samples were drawn. The test data were fixed. Uncertainty was separated into two types: variability and bias. The calculation steps of the bootstrap pairs sampling algorithm are as follows [18]:

1) Generate $J$ samples ( $J=100$ in this paper) from the development data, each one of size $N$ that was drawn with replacement from the $N$ training data $\left\{\left(\mathbf{x}_{1}, y_{1}\right),\left(\mathbf{x}_{2}, y_{2}\right)\right.$, $\left.\ldots,\left(\mathbf{x}_{N}, y_{N}\right)\right\}$. Denote the $j$-th sample by $\left\{\left(\mathbf{x}_{1}^{j}, y_{1}^{j}\right),\left(\mathbf{x}_{2}^{j}, y_{2}^{j}\right)\right.$, $\left.\ldots,\left(\mathbf{x}_{N}^{j}, y_{N}^{j}\right)\right\}$.

2) For each bootstrap sample, an SVM regression model was obtained.

3) Estimate the variance and the bias of the $i$-th predicted value by

$$
\operatorname{Var}\left(\hat{y}_{i}\right)=\frac{1}{J-1} \sum_{j=1}^{J}\left[\hat{y}_{i}^{j}-\overline{\hat{y}}_{i}\right]^{2} \text { where } \overline{\hat{y}}_{i}=\frac{1}{J} \sum_{j=1}^{j} \hat{y}_{i}^{j}
$$




$$
\text { bias }=\left\{\frac{1}{n} \sum_{i=1}^{n} \frac{1}{J} \sum_{j=1}^{J}\left[\hat{y}_{i}^{j}-y_{i}^{j}\right]^{2}\right\}^{1 / 2}
$$

where $n$ is the number of the development data.

The pool of development data represents all of the available data, excluding the defined set of fixed test data. Since the bias estimates based on the training data can be much lower than the bias estimates that are based on an independent set of data, especially in the case of an overfit model, one should compute bias estimates based on the data pool rather than the training data. The estimate with a $95 \%$ confidence interval for an arbitrary test input $\mathbf{x}_{o}$ is

$$
\hat{y}_{0} \pm 2 \sqrt{\operatorname{Var}\left(\hat{y}_{0}\right)+\text { bias }^{2}}=\hat{y}_{0} \pm \delta
$$

\subsection{Analytic Method}

The regression model of Eq. (6) is rewritten as

$$
y_{i}=f\left(\mathbf{x}_{i}, \boldsymbol{\theta}\right)+\varepsilon_{i}
$$

where

$$
\boldsymbol{\theta}=\left[\begin{array}{llll}
\theta_{1} & \theta_{2} & \cdots & \theta_{p+1}
\end{array}\right]=\left[\begin{array}{lllll}
\beta_{1} & \beta_{2} & \cdots & \beta_{p} & b
\end{array}\right] .
$$

Here, $p$ is the number of the nonzero $\beta_{k}$ in Eq. (6), which is the same as the number of SVs. For a regression model $\left(y_{0}=f\left(\mathbf{x}_{0}, \boldsymbol{\theta}\right)+\varepsilon_{0}\right)$ of an observation $\mathbf{x}_{o}$, which is not part of the training data, the output prediction is given by

$$
\hat{y}_{0}=f\left(\mathbf{x}_{0}, \hat{\boldsymbol{\theta}}\right) .
$$

Using the Taylor series expansion of the output prediction to the first order, the output prediction can be approximated as follows:

$$
\hat{y}_{0} \approx f\left(\mathbf{x}_{0}, \boldsymbol{\theta}\right)+\mathbf{f}_{0}^{r} \cdot[\hat{\boldsymbol{\theta}}-\boldsymbol{\theta}]
$$

where

$$
\mathbf{f}_{0}^{r}=\left(\begin{array}{llll}
\frac{\partial f\left(\mathbf{x}_{0}, \boldsymbol{\theta}\right)}{\partial \theta_{1}} & \frac{\partial f\left(\mathbf{x}_{0}, \boldsymbol{\theta}\right)}{\partial \theta_{2}} & \cdots & \frac{\partial f\left(\mathbf{x}_{\mathbf{0}}, \boldsymbol{\theta}\right)}{\partial \theta_{p+1}}
\end{array}\right)
$$

Then the prediction error of the SVM model can be calculated as

$$
y_{0}-\hat{y}_{0}=\varepsilon_{0}-\mathbf{f}_{0}^{T} \cdot[\hat{\boldsymbol{\theta}}-\boldsymbol{\theta}]
$$

The variance of the prediction error is written as

$$
\operatorname{Var}\left(y_{0}-\hat{y}_{0}\right)=\operatorname{Var}\left(\varepsilon_{0}\right)+\operatorname{Var}\left(\mathbf{f}_{0}^{r} \cdot[\hat{\boldsymbol{\theta}}-\boldsymbol{\theta}]\right)
$$

where

$$
\varepsilon_{0} \sim N\left(0, \sigma^{2}\right) \text { and }[\hat{\boldsymbol{\theta}}-\boldsymbol{\theta}]=N(0, \mathbf{S})
$$

In the SVM model, since the parameter $\boldsymbol{\theta}$ is not solved explicitly, and is calculated implicitly with a standard quadratic programming technique in order to minimize the constrained risk function of Eq. (4), the variance-covariance matrix $\mathbf{S}$ is not calculated. However, if the parameter is assumed to be estimated explicitly with the well-known squared error minimization technique, the variancecovariance matrix can be estimated as follows [18]:

$$
\mathbf{S}=s^{2}\left(\mathbf{F}^{T} \mathbf{F}\right)^{-1}
$$

where

$$
\begin{aligned}
& s^{2}=\frac{1}{n-p-1} \sum_{i=1}^{n}\left(y_{i}-f\left(\mathbf{x}_{i}, \hat{\boldsymbol{\theta}}\right)\right)^{2}, \\
& \mathbf{F}=\frac{\partial \mathbf{y}}{\partial \boldsymbol{\theta}}=\left[\begin{array}{llll}
\frac{\partial \mathbf{y}}{\partial \theta_{1}} & \frac{\partial \mathbf{y}}{\partial \theta_{2}} & \cdots & \frac{\partial \mathbf{y}}{\partial \theta_{p+1}}
\end{array}\right], \\
& \frac{\partial \mathbf{y}}{\partial \theta_{i}}=\left[\begin{array}{llll}
\frac{\partial \mathbf{y}_{1}}{\partial \theta_{i}} & \frac{\partial y_{2}}{\partial \theta_{i}} & \cdots & \frac{\partial \mathbf{y}_{\mathbf{a}}}{\partial \theta_{i}}
\end{array}\right]^{r} .
\end{aligned}
$$

The matrix $\mathbf{F}$ is called the Jacobian matrix of the first order partial derivatives with respect to the parameters determined from the least squares.

The variance of the predicted output error can be estimated as follows [18]:

$$
\operatorname{Var}\left(y_{0}-\hat{y}_{0}\right) \approx \sigma^{2}+\mathbf{f}_{0}^{T} \mathbf{S} \mathbf{f}_{0} \approx s^{2}+s^{2} \mathbf{f}_{0}^{T}\left(\mathbf{F}^{T} \mathbf{F}\right)^{-1} \mathbf{f}_{0}
$$


The estimate with a $95 \%$ confidence interval is

$$
\hat{y}_{0} \pm 2 s \sqrt{1+\mathbf{f}_{0}^{T}\left(\mathbf{F}^{T} \mathbf{F}\right)^{-1} \mathbf{f}_{0}}=\hat{y}_{0} \pm \delta \text {. }
$$

\section{APPLICATION TO PPF ESTIMATION}

The proposed SVM was applied to the first fuel cycle of the YGN-3 PWR plant. The data that were used was obtained by running the MASTER (Multipurpose Analyzer for Static and Transient Effects of Reactor) [19] reactor analysis and design code. The MASTER code, which was developed by the Korea Atomic Energy Research Institute, was interfaced with the COBRA code for thermohydraulic calculations.

The data obtained from the simulations of the MASTER code comprised a total of 25541 input-output data points $\left(x_{1}, x_{2}, \ldots, x_{11}, y_{r}\right)$ or $\left(x_{1}, x_{2}, \ldots, x_{16}, y_{r}\right)$, depending on whether the SPND signals were used or not. In the OPR1000 nuclear power plants, the CPCS and the COLSS calculate the LPD in order to protect and monitor nuclear plants. The SPNDs, which are incore neutron detectors, have slow response characteristics. The CPCS should calculate safety-critical parameters faster than the COLSS, and thus, can protect a nuclear reactor safely from sudden accidents. Therefore, the CPCS cannot use the SPND signals, while the COLSS can use the SPND signals. Since the proposed estimation models can be applied to protection and monitoring systems, two cases were considered, one in which the SPND signals were utilized and one in which they were not. When the SPND signals are not used, the proposed algorithm can be utilized as a protection algorithm. $x_{1}$ through $x_{16}$ represent the reactor power, core inlet temperature, coolant pressure, mass flowrate, ASI, R1, R2, R3, R4, R5, P control rod positions, and 5 SPND signals (at the 5 axial levels of the center core). ASI can also be written as $\left(P_{B}-P\right) /\left(P_{B}+P_{T}\right)$, where $P_{B}$ is the bottom-half power of the nuclear reactor, and $P_{T}$ is the top-half power. $y_{r}$ is the PPF in the reactor core. R1 through R5 and P are the names of the control rod groups.

The ranges of the input and output signals that were used for training in this paper are described in Table 1. Two SVMs are optimized for two kinds of data sets, the positive (relatively high power at the top part of the reactor core) ASI cases (12765 data points) and the negative ASI cases (12776 data points). This results in smaller errors compared to using only one summed data set All of the acquired data of the positive or negative ASI cases were divided into a training data set, a verification data set, and a test data set. The test data were selected every fixed data interval (50 fixed data intervals). The test data set comprised of 256 data points.

The training data were selected by using a Subtractive Clustering (SC) scheme [20] after the test data were removed from the pool of acquired data. A SVM regression model can be optimized well by using informative data. Since the nuclear reactor system is very complex and the acquired data should cover the entire range of the operating conditions, it was expected that the input and output

Table 1. Input and Output Signal Ranges [11]

\begin{tabular}{|c|c|c|}
\hline Input signals & Nominal values & Ranges \\
\hline Reactor power (\%) & $100 \%$ & $80 \sim 103$ \\
\hline Inlet temperature $\left({ }^{\circ} \mathrm{C}\right)$ & 295.8 & $290.5 \sim 301.7$ \\
\hline Pressure (bar) & 155.17 & $131.0 \sim 160.0$ \\
\hline Mass flowrate $\left(\mathrm{kg} / \mathrm{m}^{2}-\mathrm{sec}\right)$ & 3565.0 & $2994.6 \sim 4135.4$ \\
\hline Axial shape index & - & $0.597 \sim-0.534$ \\
\hline $\mathrm{R} 1$ control rod positions $(\mathrm{cm})$ & - & $0 \sim 381$ \\
\hline $\mathrm{R} 2$ control rod positions $(\mathrm{cm})$ & - & $0 \sim 381$ \\
\hline $\mathrm{R} 3$ control rod positions $(\mathrm{cm})$ & - & $0 \sim 381$ \\
\hline $\mathrm{R} 4$ control rod positions $(\mathrm{cm})$ & - & $0 \sim 381$ \\
\hline R5 control rod positions $(\mathrm{cm})$ & - & $0 \sim 381$ \\
\hline P control rod positions $(\mathrm{cm})$ & - & $0 \sim 381$ \\
\hline SPND signals ( 3 axial positions of a core center) & - & $7.4 \sim 322.0$ \\
\hline Output signal & Nominal value & Range \\
\hline Power peaking factor & - & $1.930 \sim 4.066$ \\
\hline
\end{tabular}


training data had a lot of clusters and the data at these cluster centers were more informative than the neighboring data. The 100 training data samples were selected by changing the radius of the $\mathrm{SC}$ scheme randomly in a specified range. The cluster centers for each data sample

Table 2. Number of Support Vectors (SVs)

\begin{tabular}{c|c|c}
\hline Use of SPND signals & Sign of ASI & No. of SV \\
\hline \multirow{2}{*}{ No } & Negative & 350 \\
\cline { 2 - 3 } & Positive & 316 \\
\hline \multirow{2}{*}{ Yes } & Negative & 349 \\
\cline { 2 - 3 } & Positive & 346 \\
\hline
\end{tabular}

were discovered by an SC scheme and were used as the training data set. The training data set was comprised of 1000 data points among the 12765 or 12776 data points, for positive or negative ASI cases, respectively. The verification data consisted of all the remaining data after the removal of the test data. Table 2 shows the number of support vectors of the SVM regression models that were used for each data case. The SVs were selected from the 1000 training data points by the SVM algorithms.

Figure 2(a) shows the histogram of the exact (target) PPF values for 12520 verification data points that have negative ASI. Figure 2(b) shows the histogram of exact (target) PPF values for 12509 verification data points that have positive ASI. As can be seen in Fig. 2, PPF values are sparsely distributed for positive ASI data cases. Table 3 shows the calculation results when the SPND signals were not used. The Root Mean Squared (RMS) error is $0.14 \%$ and the maximum error is $1.26 \%$ for all of the

Table 3. PPF Calculation Results by the SVM Model when the SPND Signals were not used

\begin{tabular}{c|c|c|c|c|c|c|c|c|c}
\hline & \multicolumn{3}{|c|}{ Training data } & \multicolumn{2}{c|}{ All data except test data } & \multicolumn{3}{c}{ Test data } \\
\cline { 2 - 11 } & $\begin{array}{c}\text { No. of data } \\
\text { points }\end{array}$ & $\begin{array}{c}\text { Relative } \\
\text { maximum } \\
\text { error (\%) }\end{array}$ & $\begin{array}{c}\text { RMS error } \\
(\%)\end{array}$ & $\begin{array}{c}\text { No. of data } \\
\text { points }\end{array}$ & $\begin{array}{c}\text { Relative } \\
\text { maximum } \\
\text { error (\%) }\end{array}$ & $\begin{array}{c}\text { RMS error } \\
(\%)\end{array}$ & $\begin{array}{c}\text { No. of data } \\
\text { points }\end{array}$ & $\begin{array}{c}\text { Relative } \\
\text { maximum } \\
\text { error }(\%)\end{array}$ & $\begin{array}{c}\text { RMS error } \\
(\%)\end{array}$ \\
\hline Negative ASI & 1000 & 0.7943 & 0.0756 & 12520 & 1.2613 & 0.1380 & 256 & 0.5174 & 0.1367 \\
\hline Positive ASI & 1000 & 0.2560 & 0.1379 & 12509 & 0.8571 & $\mathbf{0 . 1 5 6 1}$ & 256 & 0.6971 & 0.1642 \\
\hline Total & 2000 & 0.7943 & 0.1112 & 25029 & 1.2613 & $\mathbf{0 . 1 4 7 3}$ & 512 & 0.6971 & 0.1511 \\
\hline
\end{tabular}

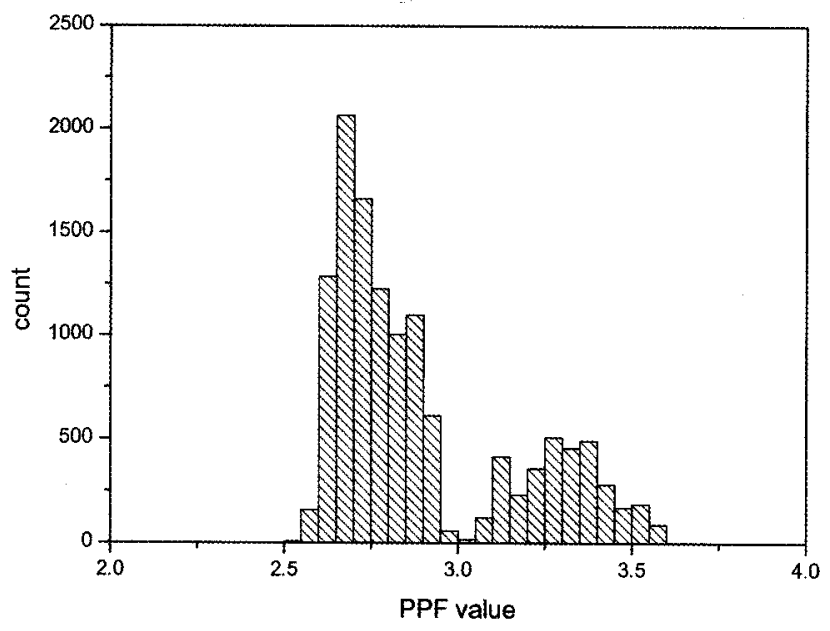

(a) histogram for negative ASI

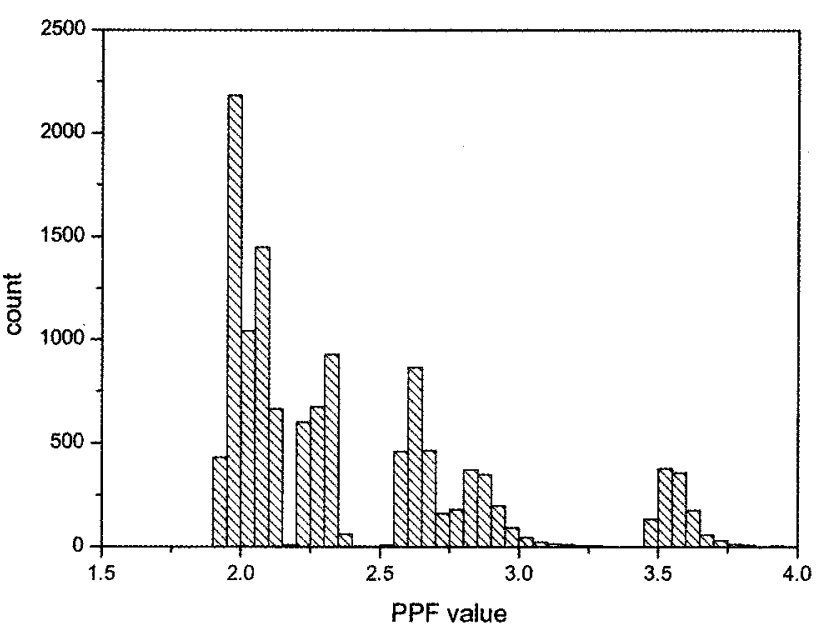

(b) histogram for positive ASI

Fig. 2. Histogram for Actual PPF Values 
data, except for the test data that have negative ASI. The RMS error is $0.16 \%$ and the maximum error is $0.86 \%$ for all of the data, except for the test data that have positive ASI. The RMS error is $0.14 \%$ and the maximum error is $0.52 \%$ for the test data that have negative ASI. The RMS error is $0.16 \%$ and the maximum error is $0.70 \%$ for the test data that have positive ASI. If we consider the relative RMS error together for both the test data sets that have positive and negative ASIs (see Table 3 ), the RMS error is $0.15 \%$.

Table 4 shows the calculation results when the SPND signals were used. The RMS error is $0.10 \%$ and the maximum error is $1.72 \%$ for all of the data, except for the test data that have negative ASI. The RMS error is $0.32 \%$ and the maximum error is $1.56 \%$ for all of the data, except for the test data that have positive ASI. The RMS error is $0.09 \%$ and the maximum error is $0.33 \%$ for the test data that have negative ASI. The RMS error is
$0.29 \%$ and the maximum error is $1.20 \%$ for the test data that have positive ASI. If we consider the relative RMS error together for both test data sets that have positive and negative ASIs (see Table 4), the RMS error is $0.21 \%$. When we used SPND signals as input to SVMs, the performance of the SVMs improved, in particular in cases that have negative ASI (refer to Tables 3 and 4). The data with positive ASI are more sparsely distributed (refer to Fig. 2), which indicates that the SPND signals do not contribute in improving the performance of SVMs for data that have positive ASI.

Figures 3 through 6 show the estimation errors and their prediction intervals. In order to conduct an uncertainty analysis by the bootstrap method, 100 sample sets for training and verification were selected by randomly adjusting the radius $r_{\alpha}$ of the $\mathrm{SC}$ scheme in a specified range. In these figures, $\delta$ indicates the prediction interval for Eqs. (10) and (19). As can be seen in Figs. 3 through

Table 4. PPF Calculation Results by the SVM Model when the SPND Signals were used

\begin{tabular}{c|c|c|c|c|c|c|c|c|c}
\hline & \multicolumn{3}{|c|}{ Training data } & \multicolumn{2}{c|}{ All data except test data } & \multicolumn{3}{c}{ Test data } \\
\cline { 2 - 10 } & $\begin{array}{c}\text { No. of data } \\
\text { points }\end{array}$ & $\begin{array}{c}\text { Relative } \\
\text { maximum } \\
\text { error (\%) }\end{array}$ & $\begin{array}{c}\text { RMS error } \\
(\%)\end{array}$ & $\begin{array}{c}\text { No. of data } \\
\text { points }\end{array}$ & $\begin{array}{c}\text { Relative } \\
\text { maximum } \\
\text { error (\%) }\end{array}$ & $\begin{array}{c}\text { RMS error } \\
(\%)\end{array}$ & $\begin{array}{c}\text { No. of data } \\
\text { points }\end{array}$ & $\begin{array}{c}\text { Relative } \\
\text { maximum } \\
\text { error }(\%)\end{array}$ & $\begin{array}{c}\text { RMS error } \\
(\%)\end{array}$ \\
\hline Negative ASI & 1000 & 0.2169 & 0.0676 & 12520 & 1.7159 & 0.0997 & 256 & 0.3275 & 0.0911 \\
\hline Positive ASI & 1000 & 0.2486 & 0.1450 & 12509 & 1.5590 & 0.3201 & 256 & 1.1957 & 0.2855 \\
\hline Total & 2000 & 0.2486 & 0.1131 & 25029 & 1.7159 & 0.2371 & 512 & 1.1957 & 0.2119 \\
\hline
\end{tabular}

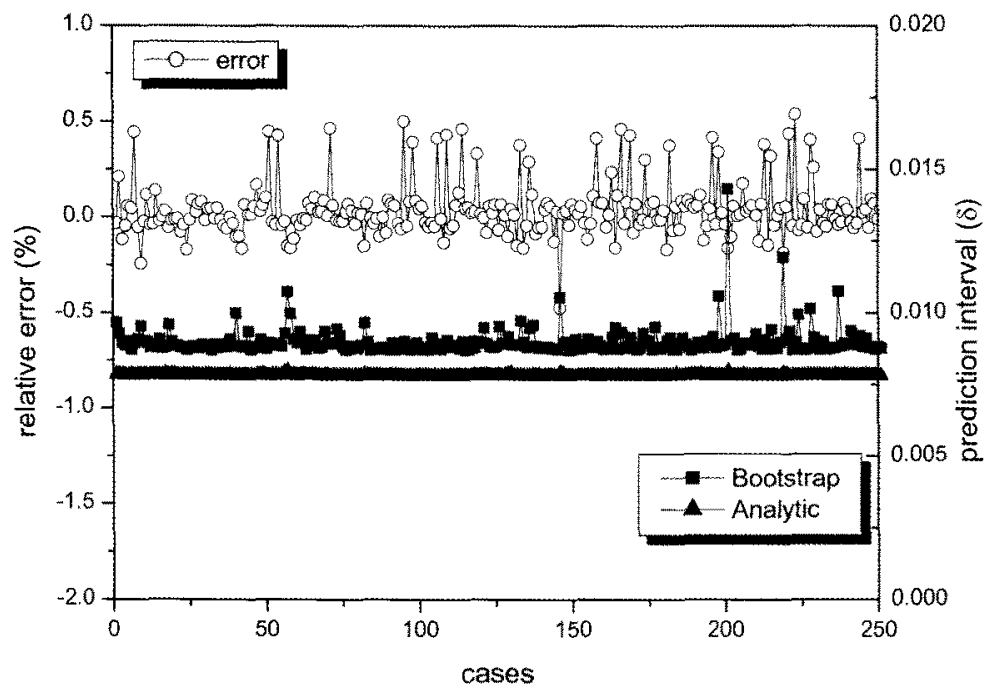

Fig. 3. Prediction Intervals of the SVM Model for Negative ASI (Without SPND Signals) 


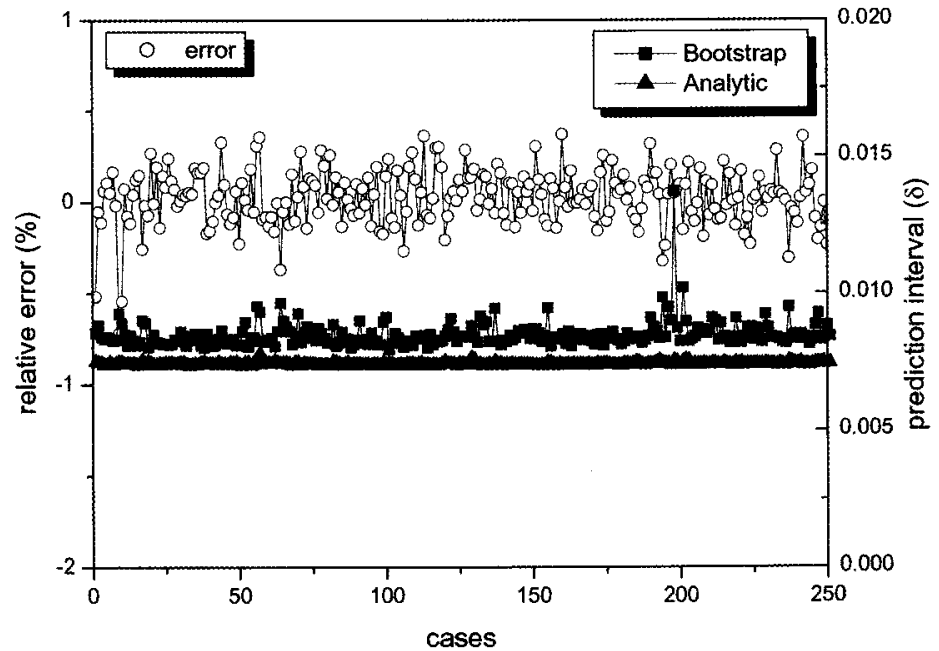

Fig. 4. Prediction Intervals of the SVM Model for Positive ASI (Without SPND Signals)

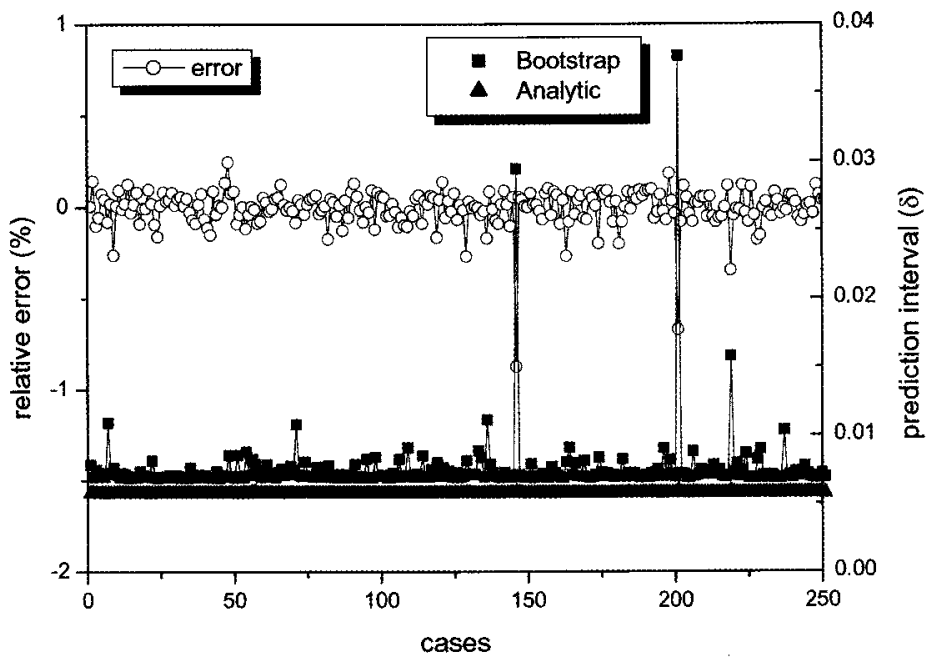

Fig. 5. Prediction Intervals of the SVM Model for Negative ASI (With SPND Signals)

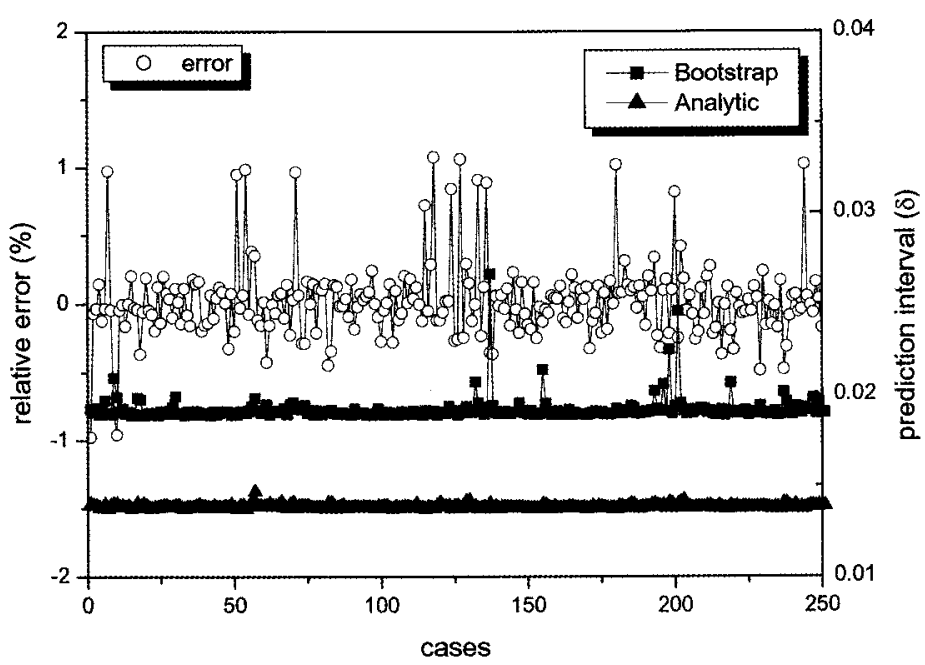

Fig. 6. Prediction Intervals of the SVM Model for Positive ASI (With SPND Signals) 
Table 5. Comparison of Calculated PPF Values

\begin{tabular}{c|c|c|c|c}
\hline ASI value & Power & MASTER (target) & SVM Model (with SPND) & COLSS \\
\hline 0.081 & 80 & 1.968 & 1.968 & 2.133 \\
\hline 0.094 & 90 & 1.959 & 1.964 & 2.135 \\
\hline 0.069 & 100 & 1.952 & 1.956 & 2.137 \\
\hline 0.073 & 103 & 1.949 & 1.953 & 2.138 \\
\hline-0.525 & 80 & 2.778 & 2.779 & 3.000 \\
\hline-0.504 & 90 & 2.718 & 2.719 & 2.961 \\
\hline-0.483 & 100 & 2.663 & 2.663 & 2.918 \\
\hline-0.520 & 103 & 2.646 & 2.646 & 2.905 \\
\hline
\end{tabular}

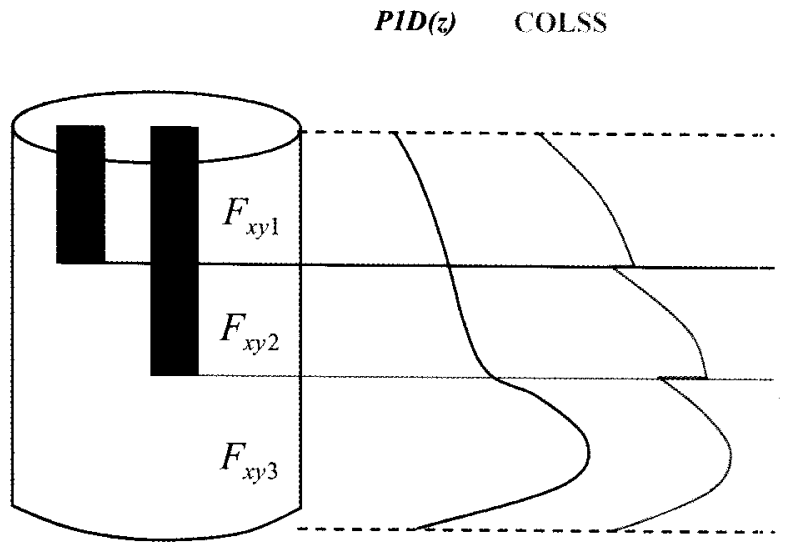

Fig. 7. Pseudo Hot Pin Axial Power Distribution of COLSS [11]

6 , the prediction intervals are very small, which means that the predicted values are very accurate. The prediction intervals of the analytic method are also similar to those of the bootstrap method. The prediction intervals of the bootstrap method can have very high peak values at several test data points because the SVM regression models that were developed by 100 random sample training data sets have a large variance at the test data points.

Table 5 shows other test results that compare the PPF values that were calculated from the proposed SVM method and the COLSS method. The PPF values of the COLSS method were obtained by multiplying the core average axial power $P \mathrm{l} D(z)$ with the plane-wise (radial direction) peaking factor $F_{x y}$ of the corresponding regions and by then selecting the maximum value of the multiplication (refer to Fig. 7). In the COLSS method, the plane-wise peaking factors are prepared and provided at the design stage according to a variety of control rod configurations. For example, for the control rod configurations of Fig. 7, each $F_{x y}$ value for 3 different regions is selected by a table lookup scheme from the $F_{x y}$ values that were prepared at the design stage. However, in the MASTER code, the plane-wise peaking factors of the real reactor core state were used to calculate the PPF value. Therefore, when the proposed SVM regression model accurately estimated the target PPF values, it always provided smaller PPF values than the COLSS method. The COLSS method is always excessively conservative when compared with the proposed SVM method, as shown in Table 5. The CPCS and the COLSS are a protection system and a monitoring system for OPR 1000 reactors (CE type plants), respectively. The CPC algorithm is more conservative than the COLSS algorithm. Therefore, the PPF values of the CPCS are larger than those of COLSS. The PPF values of the proposed algorithm were compared with those of the COLSS and it is confirmed that the PPF values of the proposed algorithm were smaller than those of COLSS, which means that the proposed algorithm provides a higher operation margin than the CPCS as well as the COLSS.

It is well-known that the RMS error that was calculated by the SVM regression model for the test data is similar to the RMS error for the verification data (see Tables 3 and 4). Therefore, if the SVM regression models are first optimized by using data for a variety of operating conditions, they can accurately estimate PPFs for other operating conditions.

\section{CONCLUSIONS}

In this paper, the SVM regression models were developed and applied in order to estimate the PPF in the reactor core. The SVM regression models were optimized by using the data set that was prepared as training data and tested by using another data set (test data) that was different from the training data. Two SVM regression models were also optimized for two kinds of data sets that were divided into both positive ASI and negative ASI, respectively. The developed SVMs were applied to the first fuel cycle of YGN-3. The RMS error of the 
estimated PPF values was about $0.15 \%$. In addition, their uncertainty was analyzed by a bootstrap method that used 100 sampled training data sets and verification data sets and was analyzed by an analytical method. The prediction intervals were very small, which means that the predicted values are very accurate. In summary, the SVM regression models are accurate enough for use in core protection and monitoring that uses power peaking factors.

\section{REFERENCES}

[1] ABB Combustion Engineering Inc., "Overview Description of the Core Operation Limit Supervisory System (COLSS)," CEN-312-P, Revision 01-P, 1986.

[2] J. Garvey, D. Garvey, R. Seibert, J.W. Hines, "Validation of on-line monitoring techniques to nuclear plant data," Nucl. Eng. Tech., vol. 39, no. 2, pp. 149-158, Apr. 2007.

[ 3 ] G.Y. Heo, "Condition monitoring using empirical models: technical review and prospects for nuclear applications," Nucl. Eng. Tech., vol. 40, pp. 49-68, Feb. 2008.

[4] H.C. Kim and S.H. Chang, "Development of a back propagation network for one-step transient DNBR calculations," Annals of Nuclear Energy, vol. 24, no. 17, pp.1437-1446, Nov. 1997.

[ 5 ] J.K. Lee and B.S. Han, "Modeling of core protection and monitoring system for PWR nuclear power plant simulator," Annals of Nuclear Energy, vol. 25, no. 7, pp. 409-420, May 1998.

[6] S.Han, U.S. Kim, and P.H. Seong, "A methodology for benefit assessment of using in-core neutron detector signals in core protection calculator system (CPCS) for Korea standard nuclear power plants (KSNPP)," Annals of Nuclear Energy, vol. 26, no. 6, pp. 471-488, 1999.

[7] M G. Na, "Application of a genetic neuro-fuzzy logic to departure from nucleate boiling protection limit estimation," Nuclear Technology, vol. 128, pp. 327-340, Apr. 1999.

[ 8 ] M. G. Na, "DNB limit estimation using an adaptive fuzzy inference system," IEEE Trans. Nucl. Sci., vol. 47, no. 6, pp. 1948-1953, Dec. 2000.

[9] W.K. In, D.H. Hwang, Y.J. Yoo, and S.Q. Zee, "Assessment of core protection and monitoring systems for an advanced reactor SMART," Annals of Nuclear Energy, vol. 29, no. 5, pp. 609-621, Mar. 2002.

[10] G.C. Lee, W.P. Baek, and S.H. Chang, "Improved methodology for generation of axial flux shapes in digital core protection systems," Annals of Nuclear Energy, vol. 29 , no. 7, pp. 805-819, May 2002.

[11] M.G. Na, D.W. Jung, S.H. Shin, K.B. Lee, and Y.J. Lee, "Estimation of the nuclear power peaking factor using incore sensor signals," Nucl. Eng. Tech., vol. 36, no. 5, pp. 420-429, Oct. 2004.

[12] I.H. Bae, M.G. Na, Y.J. Lee, and G.C. Park, "Calculation of the power peaking factor in a nuclear reactor using support vector regression models," Annals of Nuclear Energy, vol. 35, no. 12, pp. 2200-2205, Dec. 2008.

[13] V. Vapnik, The Nature of Statistical Learning Theory. New York: Springer, 1995.

[14] S.R. Gunn, Support Vector Machines for Classification and Regression. Technical Report, University of Southampton, 1998.

[15] V. N. Vapnik, Statistical Learning Theory. New York, NY: John Wiley \& Sons, 1998.

[16] V. Kecman, Learning and Soft Computing. Cambridge, Massachusetts: MIT Press, 2001.

[17] J.W. Hines, B. Rasmussen, "Online sensor calibration monitoring uncertainty estimation," Nuclear Technology, vol. 151, pp. 281-288, Sept. 2005.

[18] R. Tibshirani, "A comparison of some error estimates for neural network models," Neural Computation, vol. 8, pp. 152-163, 1996.

[19] B.O. Cho, H.G. Joo, J.Y. Cho, S.Q. Zee, "MASTER: reactor core design and analysis code," Proceedings of 2002 Int. Conf. New Frontiers of Nuclear Technology: Reactor Physics (PHYSOR 2002), Seoul, Korea, Oct. 7-10, 2002.

[20] S.L. Chiu, "Fuzzy model identification based on cluster estimation," J. Intell. Fuzzy Systems, vol. 2, pp. 267-278, 1994.

[21] M.G. Na, I.J. Hwang, and Y.J. Lee, "Inferential sensing and monitoring for feedwater flowrate in pressurized water reactors," IEEE Trans. Nucl. Sci., vol. 53, no. 4, pp. 2335 2342, Aug. 2006. 